\title{
DNA quality and quantity from up to 16 years old post-mortem blood stored on FTA cards
}

\section{Rahikainen, Anna-Liina}

2016-04

Rahikainen, A-L , Palo , J U , de Leeuw , W , Budowle, B \& Sajantila , A 2016 , ' DNA quality and quantity from up to 16 years old post-mortem blood stored on FTA cards ' , Forensic Science International , vol. 261 , pp. 148-153 . https://doi.org/10.1016/j.forsciint.2016.02.014

http://hdl.handle.net/10138/223940

https://doi.org/10.1016/j.forsciint.2016.02.014

publishedVersion

Downloaded from Helda, University of Helsinki institutional repository.

This is an electronic reprint of the original article.

This reprint may differ from the original in pagination and typographic detail.

Please cite the original version. 


\title{
DNA quality and quantity from up to 16 years old post-mortem blood stored on FTA cards
}

\author{
Anna-Liina Rahikainen ${ }^{\mathrm{a}, *}$, Jukka U. Palo ${ }^{\mathrm{a}}$, Wiljo de Leeuw ${ }^{\mathrm{b}}$, Bruce Budowle ${ }^{\mathrm{c}, \mathrm{d}}$, \\ Antti Sajantila ${ }^{\mathrm{a}, *}$ \\ ${ }^{a}$ Laboratory of Forensic Biology, Department of Forensic Medicine, University of Helsinki, P.O. Box 40 (Kytösuontie 11) FI-00014, Finland \\ ${ }^{\mathrm{b}}$ Thermo Fisher Scientific, Stockholm, Sweden \\ ${ }^{\mathrm{c}}$ Institute of Applied Genetics, Department of Molecular and Medical Genetics, University of North Texas Health Science Center, Fort Worth, TX, USA \\ ${ }^{\mathrm{d}}$ Center of Excellence in Genomic Medicine Research (CEGMR), King Abdulaziz University, Jeddah, Saudi Arabia
}

\section{A R T I C L E I N F O}

\section{Article history:}

Received 13 November 2015

Accepted 7 February 2016

Available online 23 February 2016

\section{Keywords:}

FTA cards

DNA extraction

Quantification

Inhibition

Degradation

Forensic genetics

\begin{abstract}
A B S T R A C T
Blood samples preserved on FTA cards offer unique opportunities for genetic research. DNA recovered from these cards should be stable for long periods of time. However, it is not well established as how well the DNA stored on FTA card for substantial time periods meets the demands of forensic or genomic DNA analyses and especially so for from post-mortem (PM) samples in which the quality can vary upon initial collection. The aim of this study was to evaluate the time-dependent degradation on DNA quality and quantity extracted from up to 16 years old post-mortem bloodstained FTA cards.

Four random FTA samples from eight time points spanning 1998 to $2013(n=32)$ were collected and extracted in triplicate. The quantity and quality of the extracted DNA samples were determined with Quantifiler ${ }^{\mathbb{R}}$ Human Plus (HP) Quantification kit. Internal sample and sample-to-sample variation were evaluated by comparing recovered DNA yields. The DNA from the triplicate samplings were subsequently combined and normalized for further analysis. The practical effect of degradation on DNA quality was evaluated from normalized samples both with forensic and pharmacogenetic target markers.

Our results suggest that (1) a PM change, e.g. blood clotting prior to sampling, affects the recovered DNA yield, creating both internal and sample-to-sample variation; (2) a negative correlation between the FTA card storage time and DNA quantity $(r=-0.836$ at the 0.01 level) was observed; (3) a positive correlation ( $r=0.738$ at the level 0.01 ) was found between FTA card storage time and degradation levels. However, no inhibition was observed with the method used. The effect of degradation was manifested clearly with functional applications. Although complete STR-profiles were obtained for all samples, there was evidence of degradation manifested as decreased peak heights in the larger-sized amplicons. Lower amplification success was notable with the large $5.1 \mathrm{~kb}$ CYP2D6 gene fragment which strongly supports degradation of the stored samples.

According to our results, DNA stored on FTA cards is rather stable over a long time period. DNA extracted from this storage medium can be used as human identification purposes as the method used is sufficiently sensitive and amplicon sizes tend to be $<400 \mathrm{bp}$. However, DNA integrity was affected during storage. This effect should be taken into account depending on the intended application especially if high quality DNA and long PCR amplicons are required.
\end{abstract}

() 2016 Elsevier Ireland Ltd. All rights reserved.

\section{Introduction}

Dried blood spot technology has been used to preserve samples since the 1960 s as part of newborn screenings [1]. Filter paper

\footnotetext{
* Corresponding authors. Tel.: +358504151573.

E-mail addresses: anna-liina.rahikainen@helsinki.fi (A.-L. Rahikainen), antti.sajantila@helsinki.fi (A. Sajantila).
}

based storage methods have also gained popularity in other fields, such as microbiology [2], biological fieldwork [3], bio-banking [4,5], human genomics [6,7], epigenomics [8], pharmacogenetics $[9,10]$, and forensic investigations [11-13]. Sample preservation on filter paper enables simple and flexible sample collection that allows for facile transport and archiving at room temperature. In the 1990's chemically treated filter papers became available.

Whatman $^{\mathrm{TM}}$ Flinder's Technology Associates (FTA) Cards (GE Healthcare Bio-Sciences Corp., NJ, USA) are based on a 
chemically-treated matrix, which lyses cells from a variety of tissue sources (e.g. blood, saliva, plant tissue). Upon immediate cell lysis the released DNA is bound within the supporting material, e.g. a card. The matrix protects nucleic acids from damaging agents (e.g. nucleases, oxidative agents and bacterial growth) which serves to reduce degradation. According to the manufacturer, even 22-year-old blood samples can yield short tandem repeat (STR) profiles commonly generated in forensic genetics [14]. However, most of these studies evaluated fresh blood samples which are applied immediately on the FTA cards, although other sample types also have been reported [11,15-17]. Many of these studies have targeted forensically-relevant STR markers [18,19] or single nucleotide polymorphisms (SNPs) [20], and only few studies report the results from genome wide association studies [21]. DNA quality and quantity in PM samples with variable PM intervals (PMIs) stored on FTA paper have not tested to determine if such samples meet the demands of forensic or other genomic DNA analyses.

This study evaluates time-dependent DNA degradation and the ability to amplify STRs and a longer genomic target (i.e. the CYP2D6 gene) in PM blood samples on FTA cards stored up to 16 years. First, the internal sample variation was evaluated by comparing DNA yields obtained from three different spots of the same bloodstain sample. Sample-to-sample variation was evaluated in a similar manner. Second, the quality and quantity of recovered DNA as well as the level of inhibition associated with the DNA extracts were assessed. Finally, the effective DNA yield was evaluated by determining the amplification success of short and relatively large amplicons using forensic and pharmacogenetic genotyping applications, respectively.

\section{Materials and methods}

Blood samples stored on the FTA Gene cards were obtained from forensic autopsies performed in 1998-2013. Certified standardized procedures were implemented in all autopsy procedures, including the collection of PM blood and its application onto the FTA cards. The sampled cadavers showed variation in the peri-mortem pathophysiological condition and in the extent of PM changes. To evaluate the time-dependent DNA degradation four samples were selected at random from each of the eight evenly separated time points covering the entire storage period (Fig. 1a).

\subsection{DNA extraction and quantification}

All reagents and instruments used were from Applied Biosystems (Foster City, CA, USA), Life Technology Corporation (Carlsbad, CA, USA), unless mentioned otherwise.

FTA cards were pierced with a Harris Micro-Punch ${ }^{\mathrm{TM}}$-puncher (Ted Pella, Inc., Redding, CA, USA). Each FTA bloodstain sample was extracted in triplicate, using four ( $2.0 \mathrm{~mm}$ diameter) punches as input material for each extraction (Fig. 1b). To avoid crosscontamination, the tip of the puncher was cleaned between samples by piercing clean filter paper multiple times.

The bloodstain discs were lysed according to the manufacturer's protocol using PrepFiler Express ${ }^{\mathrm{TM}}$ Forensic DNA Extraction Kit, and DNA was extracted with the semi-automated AutoMate Express $^{\mathrm{TM}}$ Forensic DNA Extraction System. Recovered DNA was eluted to a $50 \mu \mathrm{l}$ final volume with the elution buffer provided. The quality and quantity of extracted DNA were determined with Quantifiler ${ }^{\circledR}$ HP DNA Quantification Kit using 7500 Real-Time PCR system. Data analysis was performed with the HID Real-Time PCR Analysis Software v1.2.

After quantification of each extract, the triplicate samples were pooled and re-quantified with the Quantifiler ${ }^{\circledR}$ HP kit. Based on these results, the amount of PCR template DNA was normalized to $2 \mathrm{ng}$ and $35 \mathrm{ng}$ for STR analysis and CYP2D6 whole gene amplification, respectively. For the normalization, quantification results of the small autosomal target locus of the Quantifiler ${ }^{\mathbb{R}} \mathrm{HP}$ kit was used as recommended by the manufacturer [22].

DNA degradation was assessed by the degradation index (DI) defined by the Quantifiler ${ }^{\circledR}$ HP DNA Quantification kit user's manual [22]. DI measures the amplification success ratio of a small (80 bp) and a large (214 bp) autosomal target fragment. Values of DI $>1$ indicate poorer amplification of the large fragment compared with that of the short fragment, which according to the Quantifiler ${ }^{\circledR}$ HP user's manual indicates sample degradation.

Assessment of inhibition was based on amplification success of the internal positive control (IPC) in the RT-PCR assay of the Quantifiler ${ }^{\mathbb{R}}$ HP kit. Inhibiting agents present in a sample cause lower amplification success of the IPC, shown as an upward shift in the cycle threshold $\left(C_{t}\right)$ value. Under normal conditions (no inhibition), the IPC passes the threshold around cycle 27. $C_{\mathrm{t}}$-values above 30 are considered an indication of inhibition [22].

\subsection{Evaluation of the amplification success}

To measure the practical effect of degradation, amplification success of two types of autosomal loci, differing in length, was analyzed. Amplification of short fragments was assessed with GlobalFiler ${ }^{\circledR}$, a forensic STR multiplex assay (75-444 bp amplicons), and long fragments with amplification of a $5.1 \mathrm{~kb}$ genomic CYP2D6 gene fragment.

\subsection{STR analysis}

GlobalFiler ${ }^{\circledR}$ multiplex assay, which amplifies 21 autosomal STR loci, two Y chromosomal markers (DYS391, Y indel) and one sex determining marker (Amelogenin), was performed according to the manufacturer's protocol, except that the reaction volume $(7.5 \mu \mathrm{l})$ and 25 PCR cycles used. These amplification conditions have been validated in our laboratory and are part of the accredited method. Amplified DNA fragments were separated by capillary electrophoresis using a 24-capillary 3500xL Genetic Analyzer, polymer 4 (POP-4 ${ }^{\mathrm{TM}}$ ) and HID36_POP4xl Run Module. Samples were run along with 500 LIZ $^{\mathrm{TM}}$ dye size standard. Data analysis was performed with GeneMapper ${ }^{\circledR}$ ID-X ver. 1.4 software. For allele calling, 400 relative fluorescence units (rfu) were used as a peak amplitude threshold, and the fragment sizes were called using the "Local Southern" sizing method.

The STR-data was analyzed by comparing allele peak heights observed in the FTA samples with those obtained for samples of good quality, i.e. the positive control. Peak heights of the two heterozygotic peaks were summed. For the analysis, the loci were assigned relatively evenly into four size categories based on the average amplicon size: <130 bp (D3S1358, D2S441, D19S433, D22S1045, D10S1248, D8S1179), 130-200 bp (D1S1656, vWA, TH01, D5S818), 200-300 bp (D16S539, D21S11, D13S317, D7S820, D12S391), and >300 bp (CSF1PO, TPOX, D18S51, FGA, SE33, D2S1338). For each sampling time point, the average of all peak heights observed within a size class was then compared with the average peak heights observed in two independent PCRs of the positive control (DNA Control 007).

\subsection{CYP2D6 analysis}

To evaluate the integrity of longer DNA fragments the pharmacogenetically interesting CYP2D6 gene was chosen. CYP2D6 gene is part of the large CYP2D cluster which is formed together with two pseudogenes, CYP2D7 and CYP2D8P [23]. To exclude the highly homologous pseudogenes from the target of interest, the 
a)

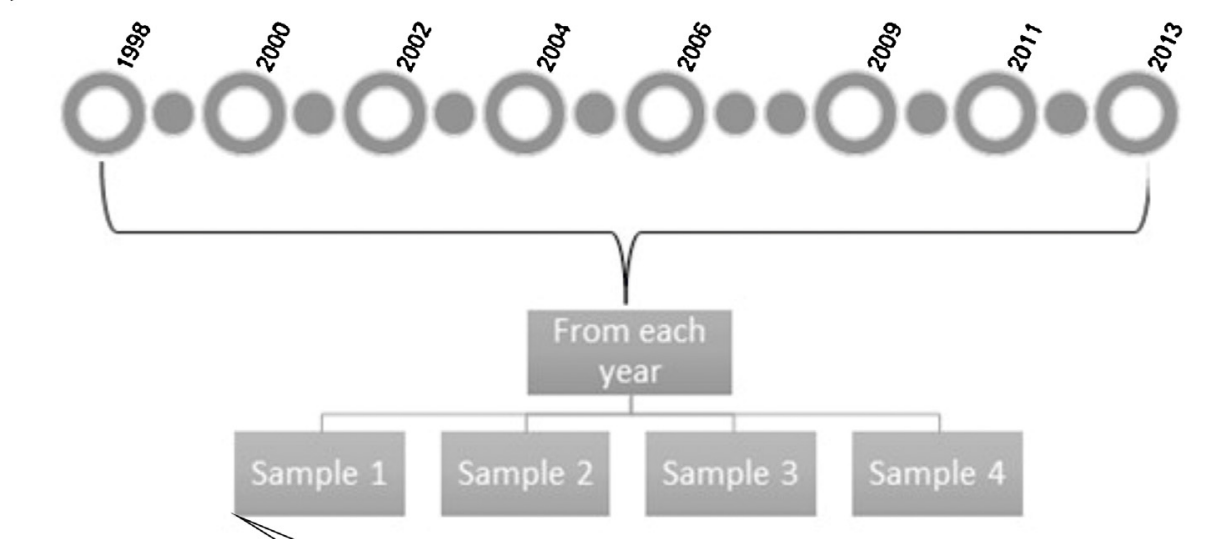

b)
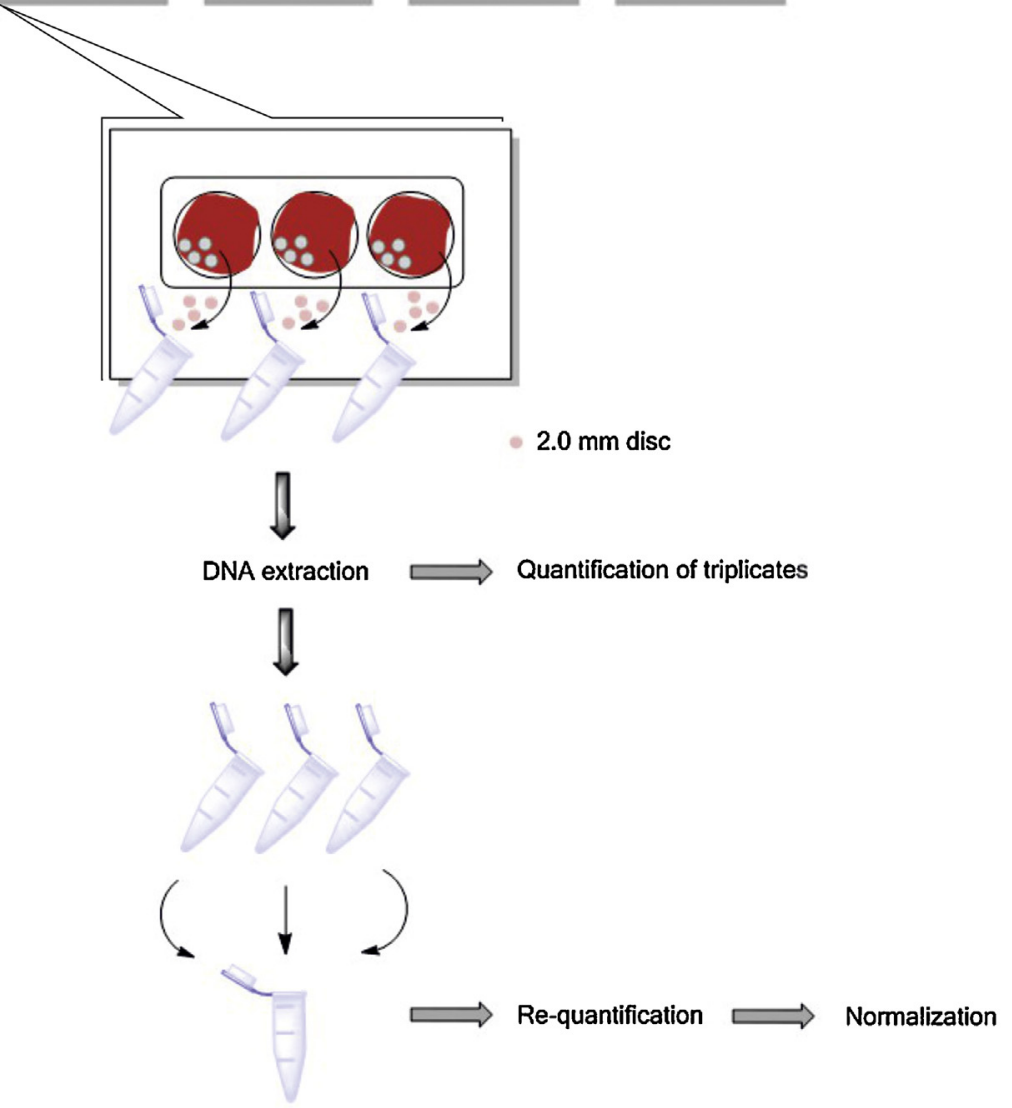

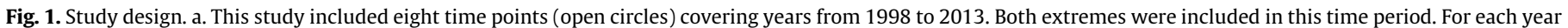

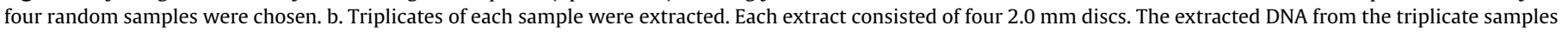
were quantified, subsequently pooled into one tube and re-quantified.

CYP2D6 gene (5.1 kb) was amplified using a previously described protocol [24] with slight modifications to the reaction mixture. Primer sequences used in this reaction were described by Lundqvist et al. [25]. The $25 \mu \mathrm{l}$ reaction mixture included $1.0 \mathrm{U}$ Platinum $^{\circledR}$ Taq DNA Polymerase High Fidelity (Invitrogen), $1 \mathrm{X}$ High Fidelity PCR Buffer, 2 mM MgSO 4 , 0.2 mM each deoxynucleotide triphosphate, $0.4 \mu \mathrm{M}$ each primer and $35 \mathrm{ng}$ of genomic DNA. Cycling conditions were the same as those described by Sistonen et al. [24]. Amplification success of the CYP2D6 gene was verified with the 2100 Bioanalyzer Instrument using the DNA 7500 kit and 2100 Expert Software for the data analysis (Agilent Technologies).

\subsection{Statistical analyses}

To measure the effect of storage time on DNA yield, harmonic means of DNA concentrations from each year were plotted against the storage time. As with the DNA concentrations, the DI values were plotted against the storage time. DI values also were plotted against PMIs. With all variables the statistical significance of correlation was assessed using Pearson Correlation Coefficient. All statistical analyses were performed with IBM SPSS Statistics ver. 22 (IMB corp. 2013, Armonk, NY, USA) software.

\section{Results and discussion}

Generally, our study confirms the previously established observations of robust and reliable DNA recovery from blood samples stored on FTA for long time periods [14]. In addition, DNA recovery from PM blood samples, with variable initial PMIs, is feasible from samples stored on FTA cards. However, our study indicates that genomic studies which require large amounts of well-preserved DNA may not be possible with long term FTA stored 
blood samples. The quality and quantity of the DNA recovered decreased over time to the extent that large-amplicon targets may no longer be amplified. Chemical treatment within the FTA cards [26] and dry storage conditions may not be sufficient for maintaining the integrity of relatively long DNA fragments.

\subsection{Internal and sample-to-sample variation; sample triplicates}

The DNA concentration ranged from 1.50 to $43.64 \mathrm{ng} / \mu \mathrm{l}$ with a mean of $10.95 \pm 8.19 \mathrm{ng} / \mu \mathrm{l}$ (Table 1 ). The amount of the input FTAmaterial was equal (four $2 \mathrm{~mm}$ punches/extraction), and each sample was extracted in triplicate. Yet variation was observed in DNA concentrations both within and among the samples. Some variation is expected with any sample manipulation. However, as the extraction method used is proven to be rather effective [12,13], high internal variation among triplicates (SD values between 0.26 to 16.07) suggests that PM changes, e.g. blood clotting prior to sampling as well as ante-mortem pathophysiological conditions of the deceased, can affect DNA recovery. Many factors, such as hemoglobin [10], hematocrit and viscosity of blood [27] and an individual's white blood cell count at the time of sampling [28] create variability in bloodstain samples. It is expected that, e.g. viscosity of blood will change post-mortem. While the intention of taking multiple punches for extraction was to reduce sampling variance, it is recommended to ensure sufficient DNA yield that multiple bloodstain discs be used per extraction (at least with the $2 \mathrm{~mm}$ diameter punches used in this study).

\subsection{Storage time and DNA quantity and quality; pooled samples}

Re-quantification results of pooled samples were similar to the mean concentration of the corresponding triplicates (data not shown) except for one sample (sample 2, 2004) where concentration of the pooled sample was lower than what was expected based of the mean concentration of triplicates. The reason for this observation is unknown, but pipetting error may be a plausible explanation.

Quantification results from the pooled samples indicate that (1) the DNA concentration is diminished over storage time; and (2)
Table 1

Internal variation of DNA concentrations extracted from bloodstained FTA cards.

\begin{tabular}{|c|c|c|c|c|c|}
\hline Year & Sample & $\begin{array}{l}\text { Mean of triplicates } \\
(\mathrm{ng} / \mu \mathrm{l})\end{array}$ & $\begin{array}{l}\text { Min } \\
(\mathrm{ng} / \mu \mathrm{l})\end{array}$ & $\begin{array}{l}\operatorname{Max} \\
(n g / \mu l)\end{array}$ & SD \\
\hline \multirow[t]{4}{*}{1998} & 1 & 1.96 & 1.50 & 2.30 & 0.42 \\
\hline & 2 & 8.46 & 7.29 & 10.75 & 1.98 \\
\hline & 3 & 5.31 & 5.05 & 5.62 & 0.29 \\
\hline & 4 & 3.74 & 2.72 & 5.67 & 1.68 \\
\hline \multirow[t]{4}{*}{2000} & 1 & 3.96 & 3.28 & 5.21 & 1.09 \\
\hline & 2 & 25.95 & 21.26 & 34.78 & 7.65 \\
\hline & 3 & 11.44 & 11.04 & 11.69 & 0.35 \\
\hline & 4 & 5.40 & 5.12 & 5.64 & 0.26 \\
\hline \multirow[t]{4}{*}{2002} & 1 & 2.92 & 2.04 & 3.51 & 0.78 \\
\hline & 2 & 7.60 & 6.15 & 8.69 & 1.31 \\
\hline & 3 & 6.20 & 5.24 & 7.41 & 1.11 \\
\hline & $4^{\mathrm{a}}$ & 6.56 & 6.12 & 7.01 & 0.63 \\
\hline \multirow[t]{4}{*}{2004} & 1 & 5.17 & 4.43 & 5.64 & 0.65 \\
\hline & 2 & 24.51 & 23.96 & 25.12 & 0.58 \\
\hline & 3 & 6.70 & 4.83 & 8.46 & 1.81 \\
\hline & 4 & 12.02 & 9.62 & 14.10 & 2.26 \\
\hline \multirow[t]{4}{*}{2006} & 1 & 6.91 & 5.48 & 7.76 & 1.25 \\
\hline & 2 & 7.10 & 3.14 & 13.49 & 5.58 \\
\hline & 3 & 19.59 & 16.91 & 21.08 & 2.32 \\
\hline & 4 & 8.04 & 7.08 & 8.56 & 0.83 \\
\hline \multirow[t]{4}{*}{2009} & 1 & 3.75 & 3.22 & 4.29 & 0.53 \\
\hline & 2 & 26.77 & 25.37 & 28.71 & 1.74 \\
\hline & 3 & 4.17 & 3.74 & 4.91 & 0.64 \\
\hline & 4 & 11.86 & 10.07 & 13.68 & 1.80 \\
\hline \multirow[t]{4}{*}{2011} & 1 & 7.14 & 6.78 & 7.45 & 0.34 \\
\hline & 2 & 18.98 & 12.93 & 22.47 & 5.26 \\
\hline & 3 & 5.10 & 3.82 & 6.34 & 1.26 \\
\hline & 4 & 25.83 & 12.44 & 43.64 & 16.07 \\
\hline \multirow[t]{4}{*}{2013} & 1 & 20.72 & 19.07 & 23.35 & 2.30 \\
\hline & 2 & 19.72 & 15.00 & 22.98 & 4.18 \\
\hline & 3 & 12.42 & 9.06 & 16.71 & 3.91 \\
\hline & 4 & 13.12 & 11.31 & 14.93 & 1.81 \\
\hline
\end{tabular}

a One of the parallel samples was excluded from the quantification measurement, because the sample evaporated from the tube due to cap malformation. Results are from two parallel samples instead of three.

DNA degradation is increased over storage time (Fig. 2). These observations were evaluated with the Pearson correlation coefficient. A significant negative correlation was observed between FTA storage time and DNA quantity at the 0.01 level $(r=-0.836)$, and a

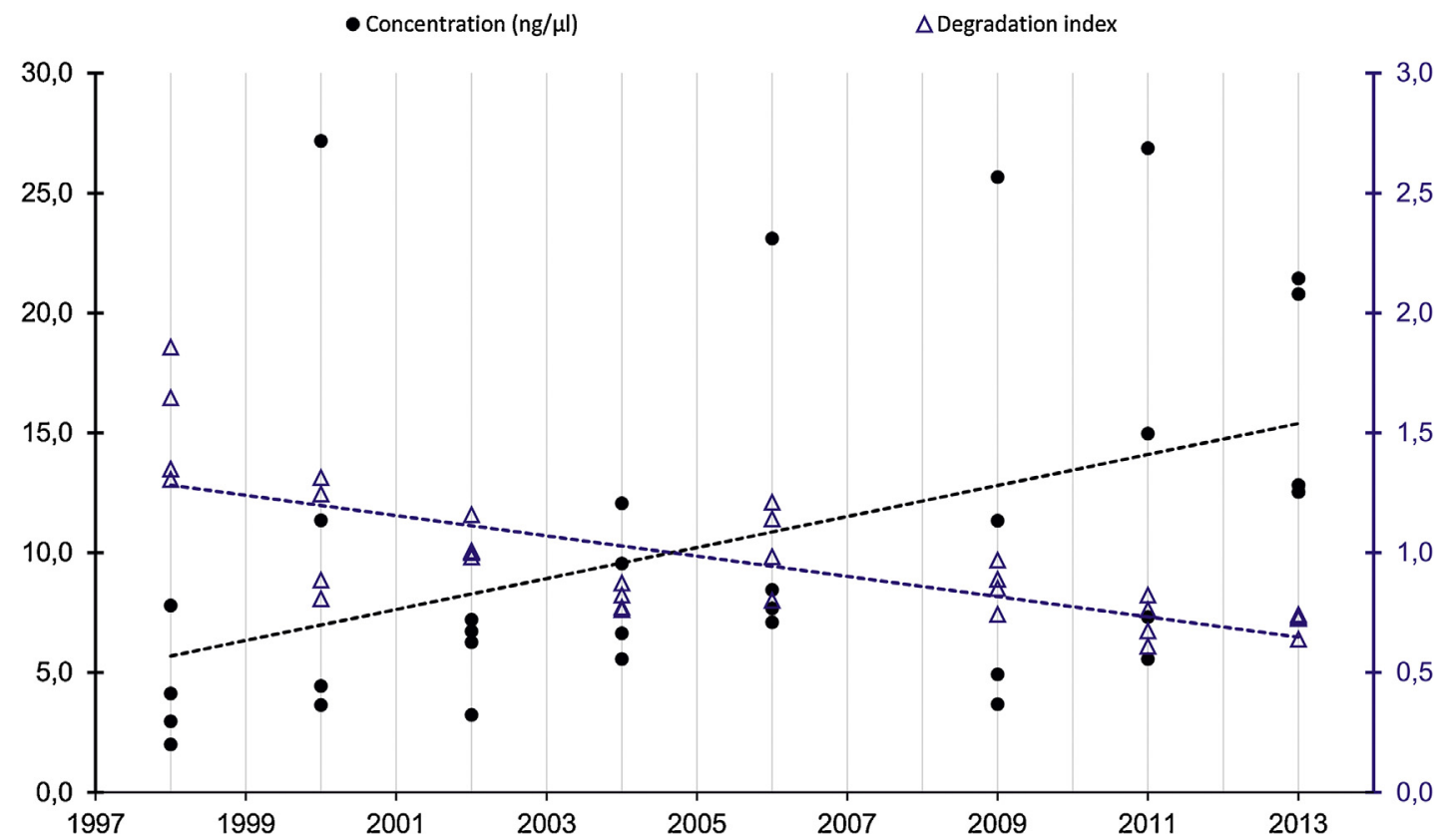

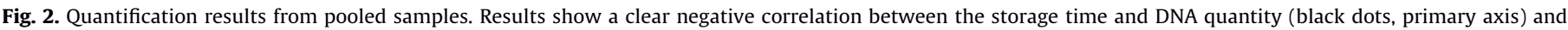
positive correlation between storage time and degradation level (open triangles, secondary axis). 
significant positive correlation ( $r=0.738$ at the level 0.01 ) between FTA storage time and degradation level. The mean value for the DI was $0.97 \pm 0.29$. To highlight the decreasing trend of sample concentrations the effect of outliers was diminished by using harmonic means of DNA concentrations.

The effect of variable PMIs to DI was evaluated, but no correlation between these parameters was observed (data not shown). As the longest PMI in this study was only 13 days, it is likely that the environmental conditions during the PMI affected DNA integrity more than the PMI time itself. However, when the PMI exceeds the 13 days the time could become a factor; but this effect could not be investigated with the current samples. In addition, no inhibition (mean $C_{t^{-}}$value $27.70 \pm 0.36$ ) was observed in this study (data not shown). Samples with high DNA concentration show a slight increase in $C_{t}$-values. However, this observation is more likely attributed to competition for reagents between the sample and the IPC than to inhibiting factors. Although in general IPC gives a rather good indication of possible PCR inhibition, normal $\mathrm{C}_{\mathrm{t}}$-values of IPC around 27 cycles cannot totally exclude possible inhibition in extracts by PCR inhibitors not affecting IPC $C_{t}$-values.

\subsection{Effects of degradation in STR and CYP2D6 analysis}

The effect of storage time on DNA degradation was clearly visible in the functional studies of STR and CYP2D6 gene analyses. The 21-loci STR profiles demonstrate clearly an increasing trend of degradation towards the older samples (Fig. 3). The shorter fragments amplify better than the longer fragments in samples of all ages, but the differences between the amplification success of the short to long fragments diminishes substantially with shorter storage times (ranging from 56 to 7\% units between 1998 and 2013, respectively). This can be taken as a clear indication of DNA fragmentation over time. Interestingly, the relative amplification success of the shorter fragment is better in the old than in the more recently stored samples. This observation could be explained by diminished competition of reagents (nucleotides, polymerase) during the PCR in absence of longer fragments. Notably, however, full STR-profiles were obtained from all samples despite the decreasing peak height with storage time.

In contrast, amplification of the larger $5.1 \mathrm{~kb}$ autosomal fragment could not be accomplished for some samples (those with a DI >1) (Fig. 4). In some cases the amplification failed even

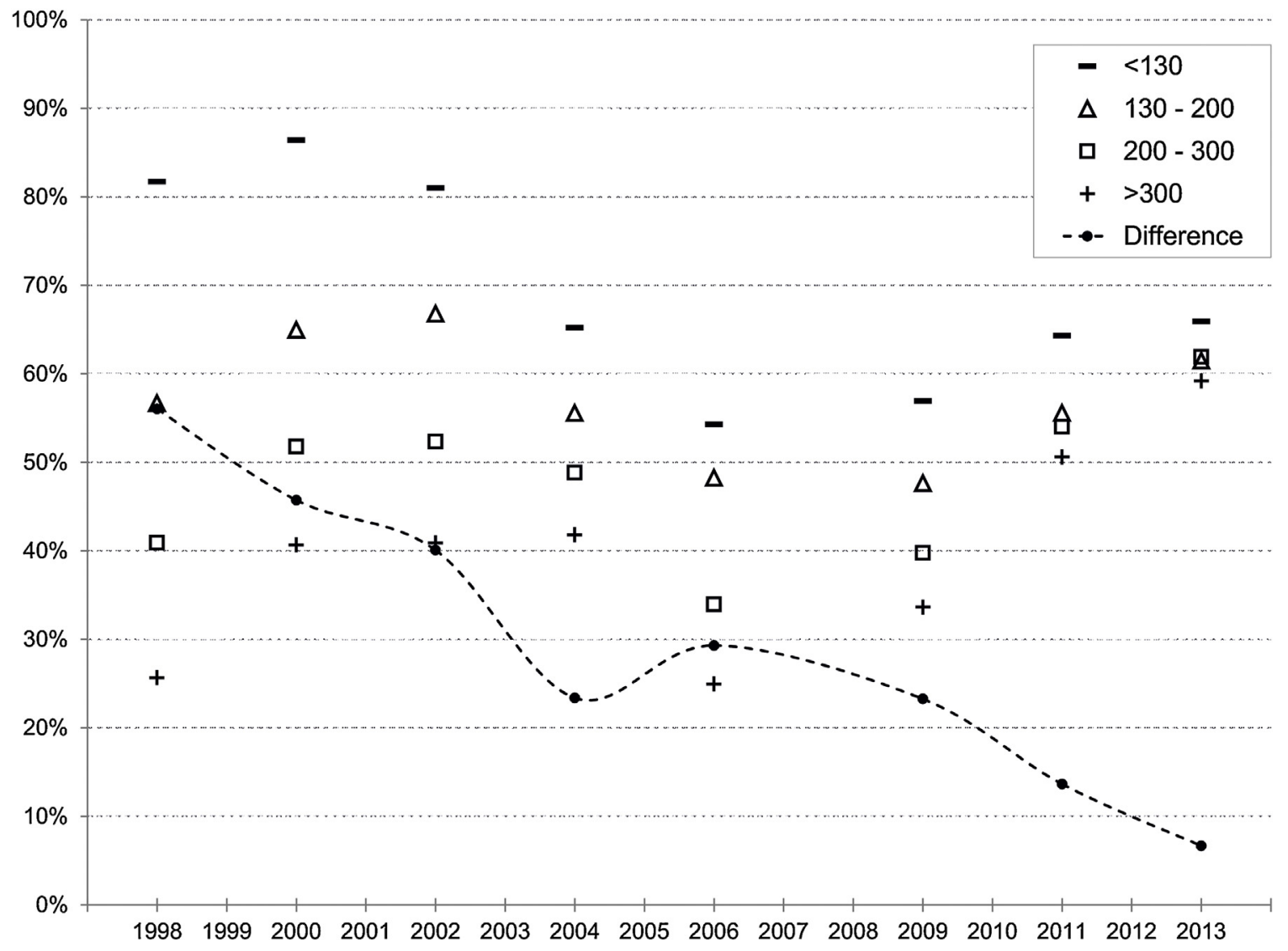

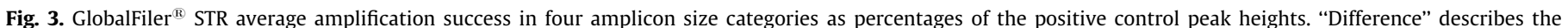

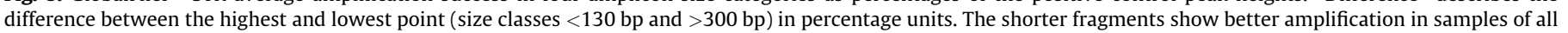

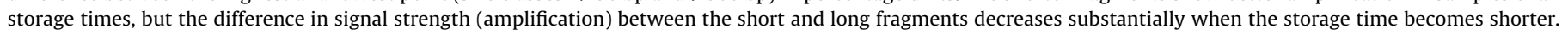

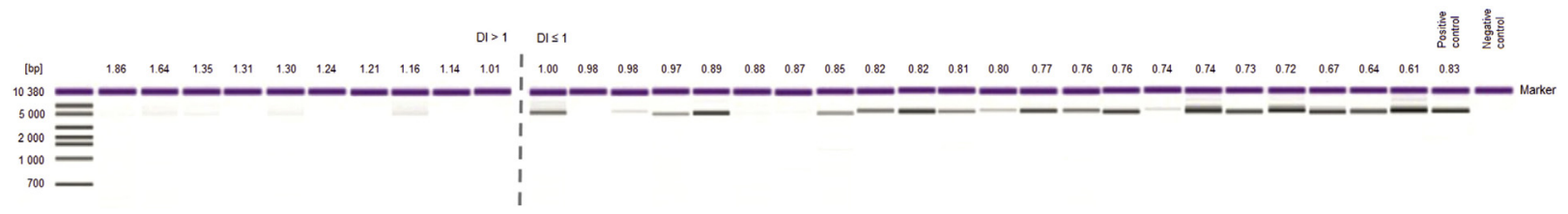

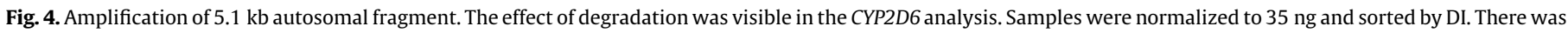
a clear difference in amplification success in the samples with DI $\leq 1$ compared to the samples with DI $>1$ (separated with dashed line). 
for samples with DI $<1$. This finding is likely due to the large size difference between the longest amplicon of the Quantifiler ${ }^{\circledR}$ HP-kit (214 bp) and the $5.1 \mathrm{~kb}$ autosomal fragment. In the case of the $5.1 \mathrm{~kb}$ fragment tested here, the general guideline of DI $>1$ as a fragmentation threshold appeared to work well, but when different size fragments are tested the threshold is likely different and should be empirically determined.

\section{Conclusions}

It is expected that PM changes prior to sampling can create notable variation in DNA quality. In addition, DNA yield may vary from sample-to-sample, depending on the white blood cell count in an individual's blood determined by the ante-mortem pathophysiological condition of the person and the PMI. As many of the factors affecting the DNA stored on the FTA cards are unknown, it is difficult, if not impossible, to predict the quality and quantity of the DNA remaining in individual samples without proper quantification.

There are some limitations to this study that should be considered. There are no control samples in the strict sense as such a study was not perceived at the time of collection of the samples especially the longest time stored samples. In order to have truly informative controls, an aliquot of the post-mortem blood sample should have been typed immediately after death (or autopsy), using the same extraction method and STR kit as for the FTA sample 16 years later. That requirement would not be feasible given the rapid development of forensic genetics techniques over the years. Any other preservation method for the sample (such as freezing) to maintain a control to compare with a FTA stored samples would also affect integrity.

As no inhibition was detected in the samples the most reasonable explanation for the amplification differences observed in this study is degradation which increases over storage time. According to our results, bloodstained FTA cards contain sufficient amounts of DNA for human identification purposes when highly sensitive STR typing is used. Obtaining sufficient intact DNA for large scale genomic studies may be more difficult. This problems created by loss of high quality DNA may be overcome, e.g. by genome wide amplification (GWA) prior to targeted PCR, as reported for large scale SNP analyses by Fowler et al. (2012) [21].

When planning experiments with relatively long amplicons, the effect of degradation must be considered and tested. Quantification indices describing concentration, degradation and inhibition provide useful tools to predict success, but the meaningful threshold values must be established specifically for different types of studies.

\section{Acknowledgments}

We would like to thank Ms. Kirsti Höök for her assistance in FTA sample collection, and also two anonymous reviewers for their valuable and constructive comments.

\section{References}

[1] R. Guthrie, Blood Screening for Phenylketonuria, J. Am. Med. Assoc. 178 (8) (1961) 863
[2] D. Rajendram, et al., Long-term storage and safe retrieval of DNA from microorganisms for molecular analysis using FTA matrix cards, J. Microbiol. Methods 67 (3) (2006) 582-592

[3] M. Muthukrishnan, et al., Evaluation of FTA cards as a laboratory and field sampling device for the detection of foot-and-mouth disease virus and serotyping by RT-PCR and real-time RT-PCR, J. Virol. Methods 151 (2) (2008) 311-316.

[4] M.I. Sjoholm, J. Dillner, J. Carlson, Assessing quality and functionality of DNA from fresh and archival dried blood spots and recommendations for quality control guidelines, Clin. Chem. 53 (8) (2007) 1401-1407.

[5] M.V. Hollegaard, et al., High-throughput genotyping on archived dried blood spot samples, Genet. Test Mol. Biomarkers 13 (2) (2009) 173-179.

[6] M.V. Hollegaard, et al., Genome-wide scans using archived neonatal dried blood spot samples, BMC Genomics 10 (2009) 297.

[7] H.S. Al Safar, et al., Evaluation of different sources of DNA for use in genome wide studies and forensic application, Appl. Microbiol. Biotechnol. 89 (3) (2011) 807-815.

[8] M.N. Cruickshank, J. Pitt, J.M. Craig, Going back to the future with Guthriepowered epigenome-wide association studies, Genome Med. 4 (10) (2012) 83.

[9] J.M. Vidal-Taboada, et al. Satisfaction survey with DNA cards method to collect genetic samples for pharmacogenetics studies, BMC Med. Genet. 7 (2006) 45.

[10] S. Mas, et al., DNA cards: determinants of DNA yield and quality in collecting genetic samples for pharmacogenetic studies, Basic Clin. Pharmacol. Toxicol. 101 (2) (2007) 132-137.

[11] Y. Fujita, S. Kubo, Application of FTA technology to extraction of sperm DNA from mixed body fluids containing semen, Leg. Med. (Tokyo) 8 (1) (2006) 43-47.

[12] C.P. Davis, et al., Extraction platform evaluations: A comparison of Automate Express $^{\mathrm{TM}}, \mathrm{EZ1} \mathbb{R}$ Advanced XL, and Maxwell $\mathbb{R} 16$ Bench-top DNA extraction systems, Legal Med. 14 (1) (2012) 36-39.

[13] M. Stangegaard, et al., Evaluation of four automated protocols for extraction of DNA from FTA cards, J. Lab Autom. 18 (5) (2013) 404-410.

[14] GE Healthcare Life Sciences. Whatman FTA Brochure; Your forensic samples, our experience. 2011 [cited 20159 Nov]; Available from: http://www.gelifesciences.com/webapp/wcs/stores/servlet/catalog/en/GELifeSciences-fi/products/ AlternativeProductStructure_21465

[15] J.M. Nechvatal, et al., Fecal collection, ambient preservation, and DNA extraction for PCR amplification of bacterial and human markers from human feces, J. Microbiol. Methods 72 (2) (2008) 124-132.

[16] G.C. Calacal, et al., Comparing different post-mortem human samples as DNA sources for downstream genotyping and identification, Forensic Sci. Int. Genet. 19 (2015) 212-220.

[17] T.L. Klassen, et al., Comparative analytical utility of DNA derived from alternative human specimens for molecular autopsy and diagnostics, J. Mol. Diagn. 14 (5) (2012) 451-457.

[18] K. Oostdik, et al., Developmental validation of the PowerPlex(R) 18D System: a rapid STR multiplex for analysis of reference samples, Forensic Sci. Int. Genet. 7 (1) (2013) 129-135.

[19] D.Y. Wang, et al., Development and validation of the AmpFISTR(R) Identifiler(R) Direct PCR Amplification Kit: a multiplex assay for the direct amplification of single-source samples, J. Forensic Sci. 56 (4) (2011) 835-845.

[20] L. Chaitanya, et al., Collaborative EDNAP exercise on the IrisPlex system for DNA-based prediction of human eye colour, Forensic Sci. Int. Genet. 11 (2014) 241-251.

[21] K.E. Fowler, et al., Novel approach for deriving genome wide SNP analysis data from archived blood spots, BMC Res. Notes 5 (2012) 503

[22] Applied Biosystems Thermo Fisher Scientific. Quantifiler $\AA$ HP and Trio DNA Quantification Kits User Guide. 2015 [cited 20159 Nov]; Available from: https://tools.thermofisher.com/content/sfs/manuals/4485354.pdf

[23] S. Kimura, et al., The human debrisoquine 4-hydroxylase (CYP2D) locus: sequence and identification of the polymorphic CYP2D6 gene, a related gene, and a pseudogene, Am. J. Hum. Genet. 45 (6) (1989) 889-904.

[24] J. Sistonen, et al., CYP2D6 genotyping by a multiplex primer extension reaction, Clin. Chem. 51 (7) (2005) 1291-1295.

[25] E. Lundqvist, I. Johansson, M. Ingelman-Sundberg, Genetic mechanisms for duplication and multiduplication of the human CYP2D6 gene and methods for detection of duplicated CYP2D6 genes, Gene 226 (2) (1999) 327-338.

[26] J. Hwang, et al., Long-term storage limits PCR-based analyses of malaria parasites in archival dried blood spots, Malar. J. 11 (2012) 339.

[27] A.J. Wilhelm, J.C. den Burger, E.L. Swart, Therapeutic drug monitoring by dried blood spot: progress to date and future directions, Clin. Pharmacokinet. 53 (11) (2014) 961-973.

[28] M.C. Kline, et al., Polymerase chain reaction amplification of DNA from aged blood stains: quantitative evaluation of the suitability for purpose of four filter papers as archival media, Anal. Chem. 74 (8) (2002) 1863-1869. 